\title{
Quantitative Risk Assessment and Application of Landslide Disaster in Chongqing
}

\author{
Ke LI ${ }^{\mathrm{a}, \mathrm{b}, 1}$, Dongsheng LIU ${ }^{\mathrm{a}}$, Yanlei WANG ${ }^{\mathrm{a}, \mathrm{b}}$ and $\mathrm{Na} \mathrm{SHE}^{\mathrm{c}}$ \\ ${ }^{a}$ Chongqing Bureau of Geology and Minerals Exploration, Chongqing 401121, China \\ ${ }^{\mathrm{b}}$ Postdoctoral Workstation of Chongqing Bureau of Geology and Mineral Exploration, \\ Chongqing 401121, China \\ ${ }^{c}$ Chongqing Energy College, Chongqing 400044, China
}

\begin{abstract}
Chongqing is located in southwestern China, and geological disasters occur frequently. The amount of potential landslide disasters is far greater than the number of landslides that can be managed by government funds, so the risk assessment for potential landslide disasters is critical. In practical applications, risk assessment methods based on landslide stability and loss are restricted by various factors. These methods can be simplified to semi-empirical assessment methods, which are influenced by the discrimination factors near the limit values of the determined conditions, possibly leading to sudden changes in the evaluation results and distort the conclusions. To solve this problem, we propose a full quantitative risk assessment method according to the probability of landslide damage. The mathematical probability model is used to quantitatively describe the risk assessment impacting factors, weaken the boundary influence, and improve the accuracy of landslide risk assessment. Correspondingly, the software is developed to conduct quantitative risk assessment on six landslides in Feng jie County, Chongqing, which verifies the accuracy and reliability of the full quantitative risk assessment method, and provides an important reference for judging urban landslide geological disasters.
\end{abstract}

Keywords. Landslide disaster, risk assessment, geological disaster

\section{Introduction}

Chongqing is located in southwestern China, and geological tragedies frequently arise. According to statistics, there are more than 14,000 potential geological disasters in Chongqing, of which more than $80 \%$ are landslides. Consequently, landslide disaster has become one of the most serious geological environmental problems in the city (figure 1). Faced with the contradiction between so many potential geological disasters and the government's limited geological disaster prevention budget, the key issue is to determine the selection rules for potential landslide geological disaster management projects.

Single landslide disaster risk investigation focuses on two elements of disaster: risk and vulnerability. Risk research includes the study of the probability of disaster damage and its influencing scope. For vulnerability investigation, it works on the disaster

1 Corresponding Author, Ke LI, Chongqing Bureau of Geology and Minerals Exploration, Chongqing 401121, China; Email: 20101601021@cqu.edu.cn. 
intensity and vulnerability on disaster-bearing bodies [1-3]. In addition, there exist two perspectives: the first viewpoint is that the stable state of the landslide is the key factor to make a choice, and the stability factor is the primary criterion for selection. The other one believes that the possible results of landslides are more important than the stability state. From the research results [4-7], it has been proved that only the risk level can be applied as a criterion for geological disaster assessment [8].

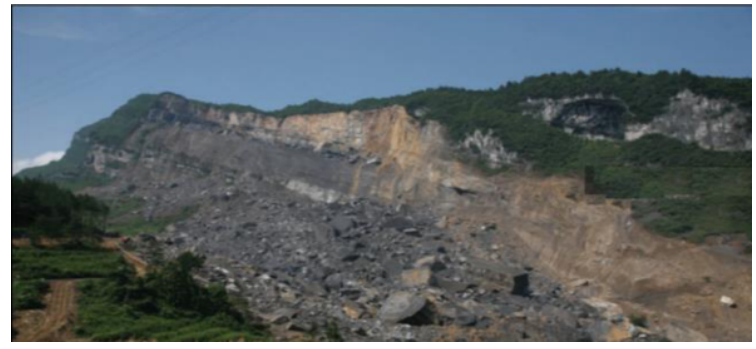

Figure 1. Landslide of Jiwei Mountain in Chongqing in 2009.

\section{Traditional Methods of Landslide Hazard Risk Assessment}

\subsection{Qualitative Risk Assessment of Landslide Disaster}

Based on the information collected from the geological survey of the landslide site, combined with the engineering experience of experts and geological engineers, the stable state of the landslide is described and the corresponding stable grade is given. The qualitative description of the stability state characteristics of the landslide generally includes the following aspects [9]: (1) Regional geological background of the landslide site; (2) The leading and triggering factors of the landslide; (3) The stage and development trend of the landslide and the possible failure modes. Meanwhile, according to "Landslide Prevention Geological Investigation Code" (GB/T 32864-2016) [10], qualitative and stable evaluation of the landslide can be performed, and the corresponding stability grades (high, medium, low) can be established Similarly, the possible damage statistics for potential landslide usually contain the following: (1) Threatening personal safety; (2) Economic losses; (3) The importance of objects threatened by landslides (both in social and economic aspect).

Based on the above statistical outcomes, the losses caused by the potential landslide can be determined and the corresponding loss grades (high, medium, low) can also be established according to "Landslide Prevention Geological Investigation Specification" (GB/T 32864-2016) [10]. Combining the stable state of the landslide and the possible losses, a risk matrix that can be used to judge the landslide disaster risk level is formed (figure 2).

\subsection{Semi-Quantitative Risk Assessment of Landslide Hazard}

To improve the accuracy of qualitative risk assessment of landslides, the value of quantitative stability factor Fs is introduced, so the corresponding risk matrix is more accurate than that of qualitative risk assessment.

Based on the stability factor Fs, the landslide classification refers to the "Landslide 
Prevention Geological Investigation Code" (GB/T 32864-2016) [10] and the specific classification criteria are shown in table 1 . With the same code and from the statistic results, the values of the protecting objects or the possible losses caused by the potential landslide can be divided into 3 grades, as shown in table 2 .

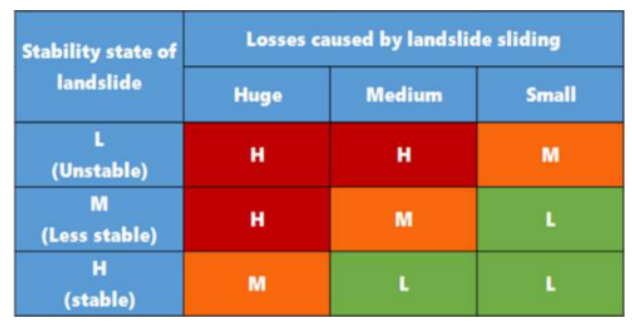

Figure 2. Risk matrix based on qualitative risk assessment of landslides.

Table 1. Classification of stable states of landslides.

\begin{tabular}{lllll}
\hline Stability factor $F_{S}$ & $\mathrm{Fs}<1.00$ & $1.00 \leq \mathrm{Fs}<1.05$ & $1.05 \leq \mathrm{Fs}<1.15$ & $1.15 \leq \mathrm{Fs}$ \\
\hline Stability state & Unstable & less stable & almost stable & stable \\
\hline
\end{tabular}

Table 2. Grade of protecting objects.

\begin{tabular}{llll}
\hline Grade of protection & $\mathrm{I}$ & $\mathrm{II}$ & III \\
\hline $\mathrm{EL}(104$ yuan $)$ & $\mathrm{EL} \geq 5000$ & $5000>\mathrm{EL} \geq 500$ & $\mathrm{EL}<500$ \\
\hline $\mathrm{TP}$ & $\mathrm{TP} \geq 500$ & $500>\mathrm{TP} \geq 100$ & $\mathrm{TP}<100$ \\
\hline $\mathrm{PI}$ & Very Important & Important & Average \\
\hline $\begin{array}{l}\mathrm{EL}=\text { Economic Loss } \\
\text { Only one condition can be defined as the corresponding protection grade. }\end{array}$ & \\
\hline
\end{tabular}

Based on the above two tables, a semi-quantitative risk matrix can be obtained to judge the risk level of landslide disasters.

Figure 3 shows that the semi-quantitative risk assessment gives specific judging standard and dividing boundaries, with the correct trend and strong application. However, due to the discontinuity of the risk interval division, there are still some problems in the application. Taking the Threatened Person (TP) of a landslide as an example, if the stable state of the landslide keeps unchanged (stability factor Fs is a constant), the change in TP can cause the risk level of the landslide to alter as follows.

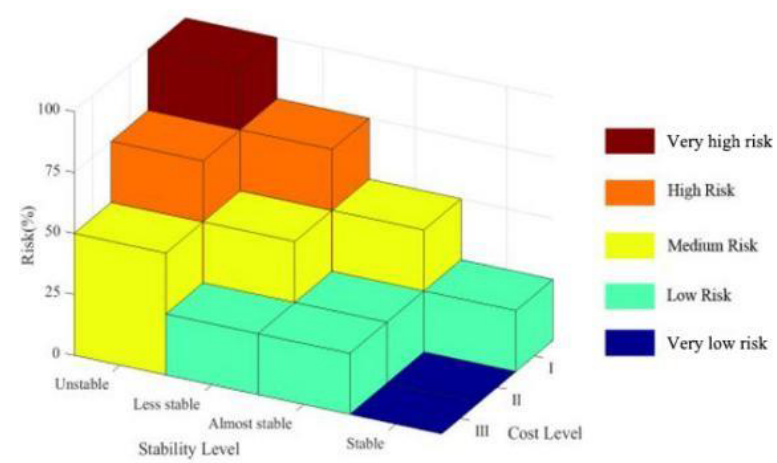

Figure 3. Spatial relationship between semi-empirical risk level and control factors. 
Table 3 shows that even if Fs remains the same, an increase in TP of 2 persons (from 99 to 101 or from 499 to 501) will change the risk level (Low to Medium or Medium to High). Conversely, under the same situation of stability, an increase in TP of 398 people (from 101 to 499) is at the same level of risk. This is obviously not a reasonable conclusion. Similarly, taking the Economic Loss (EL) as an example and keeping the landslide in the same stable state, the change in EL may generate the following alterations in the risk level of the landslide.

Table 3. Change of risk level caused be TP.

\begin{tabular}{lll}
\hline \multicolumn{2}{l}{ Stability factor: Fs $=1.04$} & Stability state: Less stable \\
\hline TP: 99 & Grade: III & Risk Level: Low \\
TP:101 & Grade: II & Risk Level: Moderate \\
TP:499 & Grade: II & Risk Level: Moderate \\
TP:501 & Grade: I & Risk Level: High \\
\hline
\end{tabular}

Table 4 shows that an increase in EL of $20 \times 10^{4}$ yuan (from $490 \times 10^{4}$ to $510 \times 10^{4}$ or from $4990 \times 10^{4}$ to $5010 \times 10^{4}$ ) changes the risk level (from Low to Medium or from Medium to High), but at the same situation of stability, an increase in TP of $4480 \times 10^{4}$ (from 510 to 4990) will not alter the risk level (from Moderate to Medium). This is an unreasonable conclusion. In addition, when the stability factor increases by 0.01 (from 1.04 to 1.05 ), the stable state will be from less stable to almost stable, while the stability factor increases by 0.1 (from 1.05 to 1.15 ), the stable state remains unchanged.

Table 4. Change of risk level caused be EL.

\begin{tabular}{llll}
\hline \multicolumn{2}{l}{ Stability factor: Fs=1.04 } & Stability state: Less stable \\
\hline EL $\left(10^{4}\right):$ & 490 & Grade: III & Risk Level: Low \\
EL $\left(10^{4}\right):$ & 510 & Grade: II & Risk Level: Moderate \\
EL $\left(10^{4}\right):$ & 4990 & Grade: II & Risk Level: Moderate \\
EL $\left(10^{4}\right):$ & 5010 & Grade: I & Risk Level: High \\
\hline
\end{tabular}

\section{Fully Quantitative Risk Assessment of Landslide}

The stable state and potential loss of the landslide must be quantitatively analyzed. Considering the variability of rock strength parameters, the stability factor should be a random variable. Also, it is more reasonable to apply the failure probability (sliding probability) to describe the stable state of the landslide.

According to the definition of landslide stability factor, $F_{s}$ can be expressed as

$$
F_{\mathrm{s}}=R / T
$$

where $R$ is the anti-sliding force, $T$ is the sliding force (DB50/5029-2004) [11].

If the relevant strength parameters of geotechnical materials are regarded as random variables with normal distribution, the corresponding stability factor $F_{s}$ is also a random variable. For convenience, assume that the stability factor $F_{s}$ is a random variable, following a normal distribution.

The $P D F$ of $F_{s}$ can be expressed as [12-14]: 


$$
f(x ; \mu, \sigma)=\frac{1}{\sigma \sqrt{2 \pi}} \exp \left(-\frac{(x-\mu)^{2}}{2 \sigma^{2}}\right)
$$

The $C D F$ (sliding probability)_of $F_{s}$ follows [15-18]:

$$
P(0<x \leq 1)=\frac{1}{\sigma \sqrt{2 \pi}} \int_{0}^{1} \exp \left[-\frac{1}{2}\left(\frac{x-\mu}{\sigma}\right)^{2}\right] d x
$$

In which, $x=F s, \mu=M_{F s}, \sigma=\Delta_{F s}=n \cdot M_{F s}$.

$M_{F s}$ is the mean of $F_{s}, \Delta_{F s}$ is the standard deviation of $F_{s}, n$ is the coefficient of $F_{s}$ variation.

Because $F_{s}$ and $n$ are related to the strength parameters of rock and soil materials, the failure probability (sliding probability) of the landslide can be defined as $P\left(F_{s} / n\right)$, suggesting the landslide failure probability is related to a mean of $F_{s}$ and a variation coefficient of $n$.

To study the change of landslide failure probability with $F_{s}$ and $n$, a comparative analysis is conducted and the partial results of calculation are shown as figure 4 .

As can be seen from figures $5 a-5 b$, the failure probability has the same coefficient of variation $(n=0.2)$ but has different means of the stability factor $\left(F_{s}=0.95\right.$ and 1.15 , respectively). Figures $5 \mathrm{c}-5 \mathrm{~d}$ show the failure probability with the same mean of stability factor $\left(F_{s}=0.95\right)$ but with dissimilar coefficients of variation $(n=0.1$ and 0.3 , respectively). Figures 5e-5f indicates the failure probability with the same mean of stability factor $(\mathrm{Fs}=1.20)$ but with different coefficients of variation ( $\mathrm{n}=0.1$ and 0.3 , respectively). These calculation results can be summarized in tables 5-7.

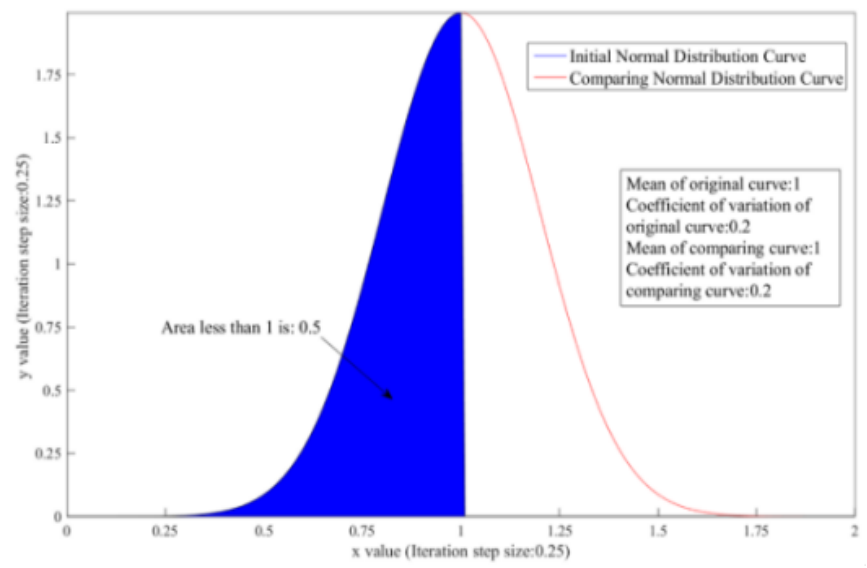

Figure 4. $\mathrm{PDF}$ and $\mathrm{CDF}$ of $\mathrm{F}_{\mathrm{s}}($ mean $=1, \mathrm{n}=0.2)$. 


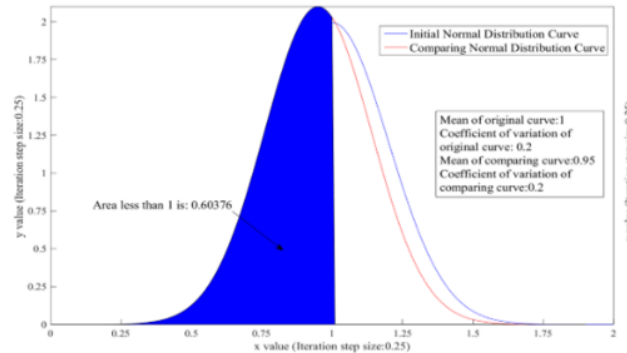

(a)

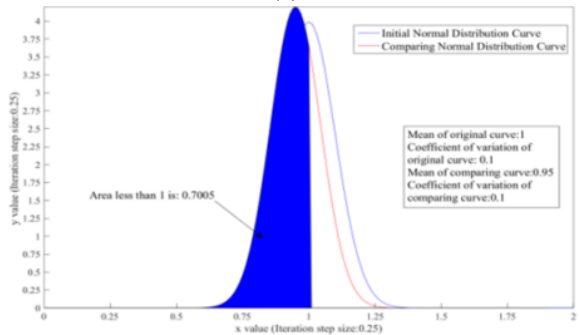

(c)

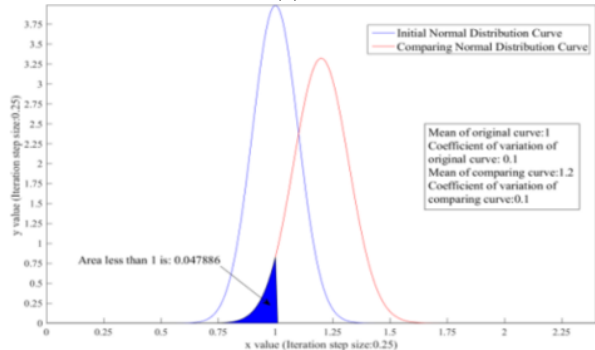

(e)

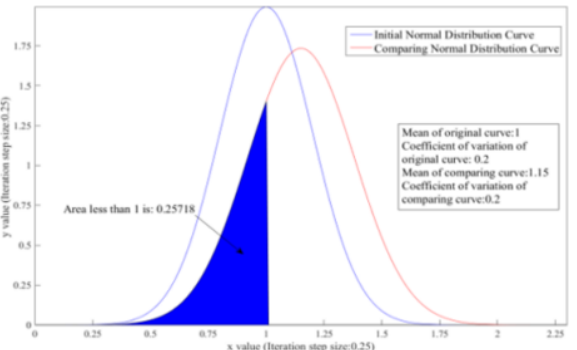

(b)

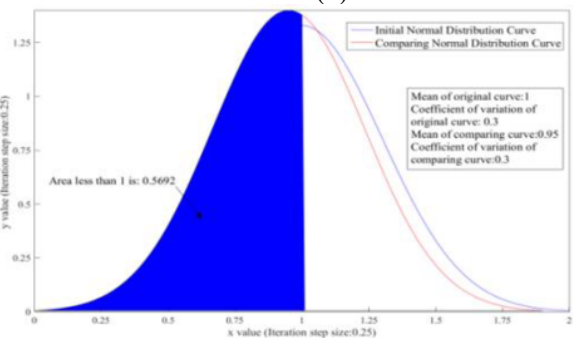

(d)

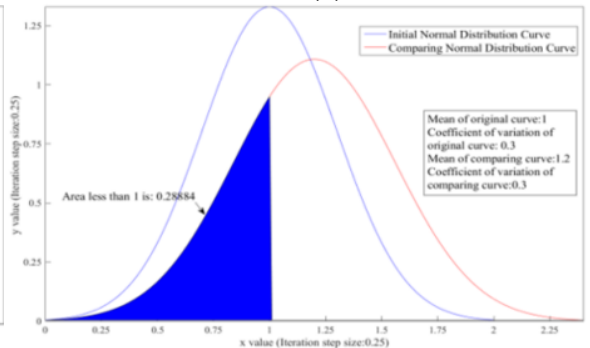

(f)

Figure 5. CDF of $\mathrm{P}(0.95 \sim 1.20 / 0.1 \sim 0.3)$ : (a) CDF of $\mathrm{P}(0.95 / 0.2)$, (b) CDF of $\mathrm{P}(1.15 / 0.2)$, (c) CDF of $\mathrm{P}$ (0.95/0.1), (d) CDF of P (0.95/0.3), (e) CDF of P (1.20/0.1), (f) CDF of P (1.20/0.3).

Table 5. Variation range of failure probability with the stability factor $F_{s}(n=0.2)$.

\begin{tabular}{lllll}
\hline Fs & 0.95 & 1.0 & 1.05 & 1.15 \\
\hline$n$ & 0.20 & 0.20 & 0.20 & 0.20 \\
$P$ & $60.38 \%$ & $50.00 \%$ & $40.59 \%$ & 25.71
\end{tabular}

Table 6. Variation range of failure probability with the coefficient of variation $\mathrm{n}\left(F_{s}=0.95\right)$.

\begin{tabular}{llll}
\hline$F_{S}$ & 0.95 & 0.95 & 0.95 \\
\hline$n$ & 0.10 & 0.20 & 0.30 \\
$P$ & $70.05 \%$ & $60.38 \%$ & $56.92 \%$ \\
\hline
\end{tabular}

Table 7. Variation range of failure probability with the coefficient of variation $n\left(F_{s}=1.20\right)$.

\begin{tabular}{llll}
\hline Fs & 1.20 & 1.20 & 1.20 \\
\hline$n$ & 0.10 & 0.20 & 0.30 \\
$P$ & $4.78 \%$ & $20.24 \%$ & $28.88 \%$ \\
\hline
\end{tabular}


The trend that the failure probability $P$ changes with the mean of $F_{s}$ and the coefficient of variation $n$ can be obtained, as shown in figure 6 .

Based on the calculation method of sliding probability (P) above, and considering the statistical results of potential landslide loss $(\mathrm{C})$, the risk index $(\mathrm{R})$ of landslide can be expressed as follows [19]:

$$
R=P \cdot C
$$

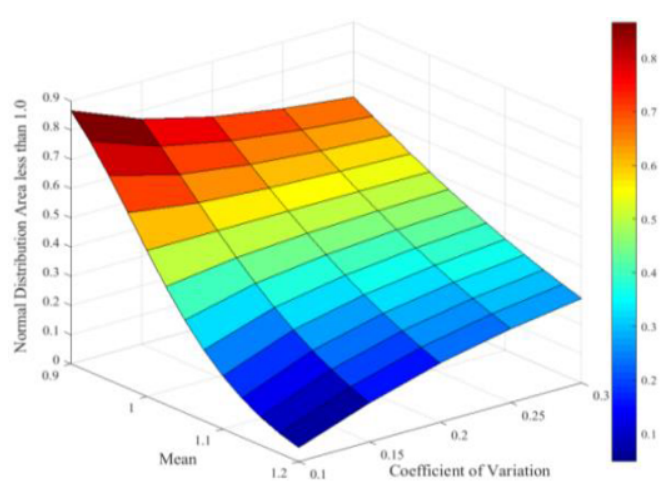

Figure 6. Trend surface of failure probability changing with mean of $F s$ and $n$.

The corresponding full quantitative risk index can be obtained, and the variation of risk index with $\mathrm{P}$ and $\mathrm{C}$ is shown in figure 7.

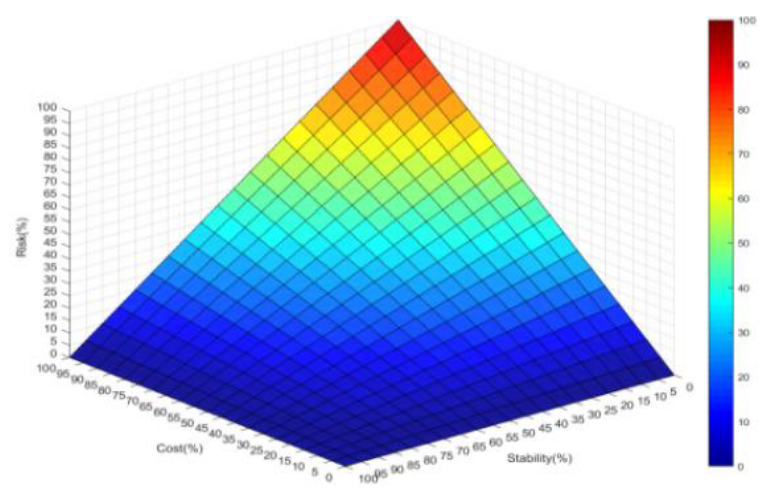

Figure 7. Spatial distribution of risk index.

\section{Engineering Applications of Landslide Assessment}

Based on the geological survey reports of six landslides in Fengjie county, Chongqing, six quantitative risk assessment reports were conducted using quantitative risk assessment software. The six corresponding indexes about stability state, threatened person (TP) and economic loss (EL) were obtained and sorted according to the index values, as shown in table 8 . It can be concluded that the results of quantitative risk assessment can provide important references for the judgment and management of urban landslide disasters. 
Table 8. TP risk levels of 6 landslides in Fengjie county, Chongqing.

\begin{tabular}{|c|c|c|c|c|c|c|}
\hline \multirow{2}{*}{ Name of landslide } & \multirow{2}{*}{ State of analysis } & \multicolumn{2}{|c|}{ Grade of Stability } & \multirow{2}{*}{$\begin{array}{l}\text { Sliding } \\
\text { probability }\end{array}$} & \multicolumn{2}{|c|}{ Risk Index of Ranking of } \\
\hline & & $\mathrm{TP}$ & state & & $\mathrm{TP}$ & TP risk \\
\hline $\begin{array}{l}\text { Chejiaba } \\
\text { landslide }\end{array}$ & Heavy rain & I & $\begin{array}{l}1.075 \\
\text { Almost } \\
\text { stable }\end{array}$ & $28.52 \%$ & 1.5899 & 1 \\
\hline $\begin{array}{l}\text { Huoshiliang } \\
\text { Landslide }\end{array}$ & Heavy rain & II & $\begin{array}{l}1.013 \\
\text { Less stable }\end{array}$ & $34.75 \%$ & 0.7536 & 2 \\
\hline $\begin{array}{l}\text { Wanjiaping } \\
\text { landslide }\end{array}$ & Heavy rain & II & $\begin{array}{l}1.037 \\
\text { Less stable }\end{array}$ & $31.18 \%$ & 0.4334 & 3 \\
\hline $\begin{array}{l}\text { Chenjiagou } \\
\text { landslide }\end{array}$ & $\begin{array}{l}\text { Heavy rain High } \\
\text { water level }\end{array}$ & II & $\begin{array}{l}1.069 \\
\text { Almost } \\
\text { stable }\end{array}$ & $29.16 \%$ & 0.4124 & 4 \\
\hline $\begin{array}{l}\text { Fangniuping } \\
\text { landslide }\end{array}$ & Heavy rain & II & $\begin{array}{l}1.065 \\
\text { Almost } \\
\text { stable }\end{array}$ & $29.34 \%$ & 0.2993 & 5 \\
\hline Laolingou landslid & Heavy rain & II & $\begin{array}{l}1.356 \\
\text { Stable }\end{array}$ & $11.77 \%$ & 0.2649 & 6 \\
\hline
\end{tabular}

\section{Conclusions}

(1) On the basis of failure probability analysis, a quantitative risk assessment method for landslide is proposed.

(2) A technical route of the assessment is established and the corresponding computer software is developed. Using this software, the quantitative risk assessment report is performed on six landslides in Fengjie county, Chongqing.

(3) With applying to the real cases, it can be concluded that quantitative risk assessment of landslide is better than qualitative or semi-quantitative risk assessment both in theoretical analysis and practical application.

(4) Quantitative risk assessment results can provide important references for the judgment of landslide disasters and play an important role in urban geological disaster management.

\section{Acknowledgements}

This work was supported by the Chongqing Natural Science Foundation Project (Postdoctoral Science Foundation) (cstc2020jcyj-bsh0137) and Scientific research project of Chongqing Bureau of Geology and Minerals Exploration (DKJ-2020DZJ-A015).

\section{References}

[1] Abedini M, Ghasemian B, Shirzadi A, et al. 2019 A novel hybrid approach of Bayesian logistic regression and its ensembles for landslide susceptibility assessment Geocarto International 34 1427-1457.

[2] Chen W, Shahabi H, Shirzadi A, et al. 2019 Novel hybrid artificial intelligence approach of bivariate statistical-methods-based kernel logistic regression classifier for landslide susceptibility modelling Bulletin of Engineering Geology and the Environment 78 4397-4419.

[3] Chen W, Sun Z and Han J 2019 Landslide susceptibility modeling using integrated ensemble weights of evidence with logistic regression and random forest models Applied Sciences-Basel 9. 
[4] Chen W, Yan X, Zhao Z, et al. 2019 Spatial prediction of landslide susceptibility using data mining-based kernel logistic regression, naive Bayes and RBFNetwork models for the Long County area (China) Bulletin of Engineering Geology and the Environment 78 247-266.

[5] Hoang N, Mehrabi M, Kalantar B, et al. 2019 Potential of hybrid evolutionary approaches for assessment of geo-hazard landslide susceptibility mapping Geomatics Natural Hazards \& Risk 10 1667-1693.

[6] Hong H, Shahabi H, Shirzadi A, et al. 2019 Landslide susceptibility assessment at the Wuning area, China: A comparison between multi-criteria decision making, bivariate statistical and machine learning methods Natural Hazards 96 173-212.

[7] Dieu T B, Tsangaratos P, Viet T N, et al. 2020 Comparing the prediction performance of a Deep Learning Neural Network model with conventional machine learning models in landslide susceptibility assessment Catena 188.

[8] Dong V D, Jaafari A, Bayat M, et al. 2020 A spatially explicit deep learning neural network model for the prediction of landslide susceptibility Catena $\mathbf{1 8 8}$.

[9] Gao W W, Gao W, Hu R L, et al. 2018 Microtremor survey and stability analysis of a soil-rock mixture landslide: a case study in Baidian town, China Landslides 15 1951-1961.

[10] China N.S.O.T.P.S.R.O. 2016 Code for Geological Investigation of Landslide Prevention (National Standard of the People's Republic of China).

[11] Chongqing E.C.S.I 2004 Code for Design of Geological Hazard Control Engineering (Chongqing Urban Rural Development Committee).

[12] Nguyen D H, Sayama T, Sassa K, et al. 2020 A coupled hydrological-geotechnical framework for forecasting shallow landslide hazard-a case study in Halong City, Vietnam Landslides.

[13] Niu H 2020 Smart safety early warning model of landslide geological hazard based on BP neural network Safety Science 123104572.

[14] Pourghasemi H R, Kornejady A, Kerle N, et al. 2020 Investigating the effects of different landslide positioning techniques, landslide partitioning approaches, and presence-absence balances on landslide susceptibility mapping Catena 187.

[15] Shafizadeh M H, Minaei M, Shahabi H, et al. 2019 Big data in Geohazard: Pattern mining and large scale analysis of landslides in Iran Earth Science Informatics 12 1-17.

[16] Sheng X S and Pan C Y 2018 Probability Theory and Mathematical Statistics (Beijing: Higher Education Press).

[17] Wu Y, Miao F, Li L, et al. 2017 Time-varying reliability analysis of Huangtupo Riverside No.2 Landslide in the Three Gorges Reservoir based on water-soil coupling Engineering Geology 226 267-276.

[18] Yan F, Zhang Q, Ye S, et al. 2019 A novel hybrid approach for landslide susceptibility mapping integrating analytical hierarchy process and normalized frequency ratio methods with the cloud model Geomorphology 327 170-187.

[19] Yang B, Yin K, Xiao T, et al. 2017 Annual variation of landslide stability under the effect of water level fluctuation and rainfall in the Three Gorges Reservoir, China Environmental Earth Sciences 76. 